\title{
Rentabilidade econômica da mineração de bitcoins e litecoins*
}

\section{Economic profitability of mining bitcoins and litecoins}

Guilherme Albuquerque Barbosa Silva' Carlo Kleber da Silva Rodrigues ${ }^{2}$
* Recebido em: 19/03/2016. Aprovado em: 05/01/2017.

1 Aluno de graduação do curso de Ciência da Computação - FATECS - UniCEUB.

2 Doutor em Engenharia de Sistemas e Computação pela Universidade Federal do Rio de Janeiro (UFRJ, 2006). Mestre em Sistemas e Computação pelo Instituto Militar de Engenharia (IME, 2000). Graduado em Engenharia Elétrica (Eletrônica e Eletrotécnica) pela Universidade Federal de Campina Grande (UFCG, 1993). Professor do Centro Universitário de Brasília (UniCEUB) nos cursos de Ciência da Computação e Engenharia de Computação.

\section{Resumo}

Este artigo tem o objetivo de analisar a rentabilidade econômica da mineração individual de criptomoedas bitcoins e litecoins. Essa análise é baseada na avaliação da métrica eficiência energética de cada criptomoeada em cinco países da América Latina, almejando-se uma margem de lucro de 0,10 USD/hora. Três variáveis são consideradas para o cálculo da métrica em comento: a taxa de processamento de hashes do equipamento, o consumo de energia elétrica do equipamento em watts e o valor do quilowatt-hora do país onde se realiza a mineração. Os resultados obtidos indicam que a criptomoeda litecoin é uma alternativa mais viável para a mineração do que a criptomoeda bitcoin. Por fim, sugestões para trabalhos futuros encerram este artigo.

Palavras-chave: Rentabilidade. Mineração. Bitcoin. Litecoin. Criptomoeda.

\begin{abstract}
This paper has the goal of analyzing the economic profitability of the individual mining process of the bitcoin and litecoin cryptocurrencies, respectively. This analysis is based on the metric named as energy efficiency, which is evaluated for each cryptocurrency in five countries in Latin America, considering a profit goal of $0,10 \mathrm{USD} /$ hour. Three variables are considered to compute the metric: the hash-processing rate of a standard hardware in a certain period of time, the electrical energy consumption, and the price of the kilowatt/hour of the region considered. The final results suggest that Litecoin is the best choice for a more profitable mining process. Lastly, future avenues are mentioned at the end of the article.
\end{abstract}

Keywords: Profitability. Bitcoin. Litecoin. Mining. Cryptocurrency. 


\section{Introdução}

Por ser o bitcoin uma criptomoeda livre de interferências cambiais de países (MURPHY, 2013), esta tem sido uma alternativa cada vez mais atrativa para a realização de transações de pagamentos eletrônicos. Corroborando esse pensamento, o mercado de bitcoins já alcançava USD 4,05 bilhões em março de 2015 (WHITE, 2015).

Porém, é preciso dizer que esse mercado é, ainda, instável. Em 2013, o bitcoin teve uma valorização de 6.000\% em apenas um ano, alcançando a marca de USD $1.250,00$ por bitcoin. Mas, no ano seguinte, perdeu 2/3 do seu valor. Atualmente, um bitcoin é cotado em USD 379,00 .

Além dessa instabilidade, o bitcoin sofre, também, com outros tipos de problemas como: a crescente demanda computacional para a realização da mineração e, consequentemente, o maior consumo de energia elétrica, resultando em um negativo impacto ambiental.

Alternativas de criptomoedas cresceram desde o ano de 2011, com destaque para aquela denominada de litecoin. Em 2013, essa criptomoeda teve um crescimento de 400\% e, em seu ápice, chegou a ser cotada em USD 48,05. Trata-se de uma alternativa que possui a característica de um processo de mineração mais rápido que aquele do bitcoin. Além disso, como ainda não existem muitas opções de hardware especializado para mineração de litecoin, cria-se uma competição mais democrática entre os possíveis mineradores, pois estes tendem a utilizar o mesmo hardware.

Ante o exposto, surge a seguinte pergunta ainda em aberta na literatura: a despeito da complexidade computacional e sob o ponto de vista da rentabilidade econômica, é mais vantajoso minerar litecoins que bitcoins?

Dentro desse contexto, o presente trabalho tem o objetivo de analisar a rentabilidade econômica da mineração individual das criptomoedas bitcoin e litecoin, respectivamente. Essa análise baseia-se na avaliação da métrica eficiência energética nos cinco países mais ricos da América Latina, almejando-se uma margem de lucro de 0,10 USD/hora. Três variáveis são consideradas para o cálculo dessa métrica: a taxa de processamento de hashes do hardware empregado, o consumo de energia elétrica em watts e o valor em dólares do quilowatt-hora.

A organização do restante desse texto é descrita a seguir. A Seção 2 apresenta, sucintamente, o protocolo Bitcoin e as suas principais diferenças com relação ao protocolo Litecoin. Os trabalhos relacionados são discor- ridos na Seção 3. Na Seção 4, são apresentados e discutidos os experimentos e os resultados alcançados. Por fim, as conclusões finais e os direcionamentos para trabalhos futuros aparecem na Seção 5.

\section{Fundamentos}

\subsection{Protocolo Bitcoin}

O protocolo Bitcoin é o resultado de um artigo publicado em novembro de 2008 sobre a ideia de uma criptomoeda, denominada de bitcoin, que funciona, de forma anônima e, principalmente, sem depender da confiança em qualquer usuário do sistema (NAKAMOTO, 2008). Satoshi é o nome dado a menor quantidade de bitcoins que pode ser enviada em uma transação: 0,00000001 bitcoin (BADEV et al., 2008). Esse protocolo é implementado sobre uma rede peer-to-peer (P2P) de alcance mundial, redundado em um eficiente sistema de transações comerciais ou pagamentos eletrônicos.

Um ponto de significativa importância é o fato de a moeda bitcoin ser descentralizada, i.e., a própria arquitetura da rede $P 2 P$ garante a autenticidade e o saldo financeiro (i.e., saldo da carteira virtual de bitcoins) de todas as pessoas envolvidas. Por isso, o bitcoin está livre de influências alfandegárias de bancos centrais de países e interferências políticas no valor da moeda (DOWD; HUTCHINSON, 2015).

A arquitetura do protocolo Bitcoin é baseada em um livro-razão ou ledger público, em que todos os usuários têm acesso a todas as transações e saldos, mas, como são utilizados números em vez de nomes, a privacidade é, então, preservada (NAKAMOTO, 2008).

Toda vez que uma transação em bitcoins é realizada, o ledger é atualizado em todos os computadores da rede. Para exemplificar, considere a transação fictícia explicada a seguir entre dois usuários imaginários: Amanda e Bruno. Amanda compra um produto de Bruno no valor de 1,0 BTC (BTC = bitcoin). Tanto o ledger de Bruno quanto o de Amanda vão ter seus saldos alterados: em mais 1,0 BTC (para Bruno) e menos 1,0 BTC (para Amanda). No entanto, o restante da rede inteira, também, tem que atualizar seus respectivos ledgers para conter os saldos atualizados de Bruno e Amanda.

\subsection{Conceito de Block-chain}

Em relação ao exemplo da subseção anterior, quando Amanda envia 1,0 BTC para Bruno, é como se 
Amanda estivesse assinando um documento público que atesta: Eu, Amanda, estou dando 1,0 BTC para Bruno. A assinatura dessa transação é realizada pelo conceito de par de chaves assimétricas, em que Amanda, como qualquer usuário da rede Bitcoin, possui duas sequências de dígitos aleatórios que formam as chaves pública e privada, respectivamente.

O protocolo Bitcoin utiliza o algoritmo ECDSA (Elliptic Curve Digital Signature Algorithm) para implementar o conceito de chaves assimétricas que, nesse caso, é um número inteiro de 256 bits de tamanho (NAKAMOTO, 2008). O conhecimento da chave privada é único e exclusivo de Amanda e, juntamente a essa chave privada, é gerada outra sequência de dígitos que formam a chave pública de Amanda (ESKANDARI et al., 2015).

Esse sistema de chaves garante a autenticidade de quem e, ainda, para quem a transação é realizada, mas não garante que Amanda não possa gastar a mesma moeda mais de uma vez ou utilizar a mesma chave daquela transação em outras transações. Dado que se decorre um tempo para propagar o ledger atualizado, Amanda poderia realizar outra transação enquanto a antiga não se propagou por toda a rede. Nesse caso, a rede teria dificuldades em diferenciar qual é a transação legítima.

A solução para o problema anteriormente descrito se dá pelo emprego do conceito de block-chain, explicado a seguir. Propagam-se as transações recém-realizadas, mas, ainda, não validadas, por toda a rede. Essas transações são então agrupadas em blocos. Cada bloco é validado pelo minerador (i.e., hardware utilizado para validação) por meio de um processo matemático de alta complexidade, envolvendo hash criptográfico (NAKAMOTO, 2008).

Após validado, o bloco é, então, adicionado a uma cadeia de blocos, que leva desde o primeiro bloco, contendo a primeira transação já realizada na história do Bitcoin, até a transação mais recentemente validada. Essa cadeia é denominada de block-chain e é a base de informação para implementar o ledger púbico.

É pouco provável que dois ou mais mineradores consigam resolver o processo matemático, citado anteriormente, no mesmo instante. Porém, prevendo essa situação, toda vez que o bloco é colocado na block-chain, ele é colocado, na verdade, em uma ramificação da block-chain original, denominada branch, e os mineradores posteriores vão, sempre, escolhendo a branch de maior tamanho (i.e., comprimento mais longo). A convergência para a branch a ser, definitivamente, aceita (i.e., aquela de maior comprimento entre todas existentes) ocorre em, aproximadamente, seis blocos ou, como cada bloco leva cerca de dez minutos, em uma hora (NIELSEN, 2013).

\subsection{Conceito de Proof-of-work}

O processo matemático a ser realizado pelo minerador está relacionado ao algoritmo hash criptográfico SHA-256 (NAKAMOTO, 2008). De forma simples, o minerador precisa descobrir um número inteiro de 4 bytes, denominado de nonce, capaz de satisfazer a uma desigualdade (inequação) expressa em função desse algoritmo.

O método de descobrimento usado pelo minerador é baseado em tentativas e a condição de desigualdade é estabelecida considerando-se um valor máximo, denominado de target difficulty, que é ajustado, consensualmente, pelos nós da rede para garantir que, em média, apenas um bloco de transações válidas seja adicionado à block-chain a cada 10 minutos.

$\mathrm{O}$ valor correto descoberto pelo minerador, ou seja, o nonce correto ou golden nonce, é a prova de trabalho, ou proof-of-work, que o cálculo iterativo (por tentativas) foi realizado e que o bloco pode ser adicionado ao block-chain (AUMASSON, 2009).

De forma sistêmica, para a adequada e eficiente operação do processo de mineração, o protocolo Bitcoin depende, naturalmente, de que muitos mineradores estejam validando blocos a todo momento, o que consome um elevado poder computacional e, consequentemente, energia elétrica.

Para incentivar os mineradores a validar os blocos, o protocolo Bitcoin prevê uma recompensa em bitcoins para quem primeiro conseguir encontrar o golden nonce. Essa recompensa é conhecida como payout (NAKAMOTO, 2008). É importante ressaltar que esse valor não é fixo, sendo alterado de tempos em tempos.

Quando o Bitcoin foi lançado, o seu payout era de 50,0 BTC. Esse valor de payout é dividido por 2 a cada 230.000 blocos minerados na rede ou, aproximadamente, a cada quatro anos, já que cada bloco leva cerca de 10 minutos para ser minerado. Esse ajuste é conhecido como halving. O Bitcoin já passou por um halving e, atualmente, cada bloco minerado é recompensado com 25,0 BTC.

\subsection{Protocolo Litecoin}

Em outubro de 2011, o protocolo Litecoin foi desenvolvido por Charles Lee, então funcionário da empre- 
sa Google. É um projeto de código aberto que, na época, foi lançado em plataformas de desenvolvimento colaborativas (BRADBURY, 2013). Esse protocolo possui o tempo de mineração de cada bloco estimado em 2,5 minutos.

Ressalta-se que a ideia do protocolo Litecoin não foi a de substituir a mineração de bitcoins, mas permitir a mineração conjunta de bitcoins e litecoins. O Litecoin, também, passa pelo evento de halving a cada quatro anos aproximadamente. Por último, o algoritmo usado pelo Litecoin, para estabelecer o processo matemático da mineração, é o Scrypt (PERCIVAL, 2009). Para fins de mera comparação, as principais características dos protocolos Litecoin e Bitcoin, respectivamente, são destacadas na Tabela 1 .

Tabela 1 - Diferenças entre Litecoin e Bitcoin.

\begin{tabular}{ccccc}
\hline Criptomoeda & $\begin{array}{c}\text { Tempo médio } \\
\text { para minerar } \\
\text { um bloco }\end{array}$ & $\begin{array}{c}\text { Algoritmo } \\
\text { de hash } \\
\text { criptográfico }\end{array}$ & $\begin{array}{c}\text { Tempo para } \\
\text { cada evento } \\
\text { de halving }\end{array}$ & $\begin{array}{c}\text { Valor atual } \\
\text { em USD }\end{array}$ \\
\hline Litecoin & 2,5 minutos & Scrypt & 4 anos & 3,37 \\
Bitcoin & $\begin{array}{c}10,0 \\
\text { minutos }\end{array}$ & SHA-256 & 4 anos & 379 \\
\hline
\end{tabular}

Fonte: Os autores.

Para encerrar, cabe mencionar que existem, basicamente, três formas de se obter criptomoedas: por meio de uma transação entre criptomoedas, adquirindo criptomoedas com dinheiro real e, por último, por meio do processo de mineração. Essa última pode ser realizada em grupos de distintos hardwares (denominados de pools) ou, ainda, por meio da mineração individual, i.e., utilizando-se um único hardware. O foco deste trabalho é a mineração individual.

\section{Trabalhos relacionados}

A rentabilidade da mineração individual de criptomoedas ainda é um tema pouco discutido na literatura. Tendo-se ciência desse relativo ineditismo, esta seção busca, alternativamente, discorrer sobre quatro dos trabalhos mais recentes da literatura que contribuem ou se relacionam, mesmo de forma indireta, com o objetivo deste trabalho.

O trabalho de Rosenfield (2011) se propõe a compreender os aspectos da rentabilidade por mineração em pools. O trabalho concluiu que, por causa da alta variância nas recompensas da mineração individual, a necessidade de mineração em pools se faz importante e não pode ser desconsiderada. dade de as criptomoedas serem mais amplamente usadas ou se tornaram dinheiro de um nicho mais específico do mercado. $O$ trabalho conclui que o Bitcoin representa um real avanço tecnológico no processamento de pagamentos, embora seja, também, verdade que o desenvolvimento de outras criptomoedas possa vir em algum momento tornar o Bitcoin obsoleto, passando este a ser reconhecido apenas como o percursor das criptomoedas.

O trabalho de Chávez e Rodrigues (2015) demonstra formas de decidir, analiticamente, quando é mais vantajoso trocar, automaticamente, de pools a fim de manter a rentabilidade considerada. O trabalho concluiu que a mineração considerando o salto entre pools é mais eficiente do que a mineração considerando apenas um único pool.

Por fim, o trabalho de Pazmiño e Rodrigues (2015) avalia o tempo de verificação de transações e, nesse contexto, propõe um esquema para a divisão da base de dados de um nó da rede bitcoin, considerando o hardware disponível localmente no usuário. Os resultados finais são, numericamente, atrativos, resultando em otimizações de até $71,42 \%$ no tempo de verificação de transação.

\section{Rentabilidade com Bitcoin e Litecoin}

Tanto o Bitcoin quanto o Litecoin garantem, atualmente, 25 moedas em seu payout. Para ambos, a target difficulty (ou, simplesmente, dificuldade), representada pela letra $D$, refere-se à complexidade para se minerar um bloco, sendo ajustada de forma que qualquer valor de tentativa para encontrar o valor de nonce tenha, sempre, a razão de $\frac{1}{2^{32} * D}$ de sucesso.

O valor atual de $D$ para o protocolo Bitcoin é 144.116.447.847 e, para o protocolo Litecoin, é 54.656. A taxa com que um hardware consegue testar valores para descobrir o golden nonce é denominada de hashrate, representada pela letra $h$.

Considerando-se, então, o tempo de mineração $t$, um hardware pode realizar um total de $h^{\star} t$ tentativas (ou hashes) para descobrir o valor do golden nonce. Ainda, a quantidade de bitcoins $Q$ a ser recebida, em um certo intervalo de tempo $t$ de mineração, pode ser estimada pela Equação 1 (ROSENFIELD, 2011).

$$
Q=\frac{h * t}{2^{32} * D} * \text { payout }(1)
$$

Propõe-se, agora, calcular a rentabilidade econômica da mineração de uma moeda para qualquer país 
conforme raciocínio apresentado a seguir. Para esse cálculo, consideram-se os gastos de energia elétrica (Despesa) e o ganho durante o período (Receita), ambos calculados em dólares.

O valor da Receita é obtido pela Equação 2 e o valor de Despesa é obtido por meio da Equação 3. Sendo assim, a rentabilidade esperada é calculada como Receita menos Despesa, dado um certo período de tempo.

Explica-se que o valor de Receita é calculado levando-se em conta o valor $Q$ de moedas recebidas durante o período de tempo de mineração $t$ (Equação 1), bem como o valor de cotação atual em dólares da criptomoeda considerada, representado por $V_{c}$.

Receita $=Q \star V_{c}(2)$

Ainda, explica-se, também, que Despesa, durante a atividade de mineração, está relacionada ao valor do kWh da região onde a mineração em si é realizada. Também se leva em conta a potência do equipamento (hardware) de mineração $P$, dada em watts, que serve para o cômputo de quanta energia o equipamento precisa utilizar em determinado período de tempo $T$, medido em horas.

\section{Despesa $=\frac{P * T}{1000} * k W h(3)$}

Considere que a rentabilidade almejada seja de USD 0,10 por hora (i.e., USD 0,10/h). Esse valor é escolhido por ser compatível com o cenário atual da atividade de mineração, observado em sites populares da Internet que reportam sobre valores estimados para esse tipo de atividade. No entanto, é preciso esclarecer que esse valor absoluto não é importante para efeito das conclusões e observações a serem alcançadas neste trabalho, pois aqui tenciona-se uma análise comparativa (relativa) e não absoluta.

Considere, ainda, que a região onde a mineração é realizada seja o país Brasil. Utilizando-se, então, as Equações 1 e 2 e, também, um equipamento (hardware) padrão de mineração de potência máxima de 100 watts, chega-se aos seguintes resultados: Despesa $=$ USD 0,0096 e Receita $=$ USD 0,1096, ambos por hora

Como mencionado, para se encontrar o golden nonce, é necessário observar a medida de hashrate do equipamento. Isso para que o valor de hashrate seja suficiente para encontrar um bloco no tempo necessário para preservar o lucro esperado ao final da mineração. É considerado um valor de hashrate mínimo ou $h_{\min }$. A Equação 4 apresenta a fórmula para obter o valor de $h_{m i n}$. Observe que essa equação é derivada a partir das Equações 1,2 e 3.

$$
h_{\min }=\frac{\text { rentabilidade } * 2^{32} * D}{t * \text { payout } * V_{c}}(4)
$$

A métrica eficiência energética EE, definida neste trabalho, é medida em hash/J e representa quantos hashes um hardware padrão precisa calcular, utilizando 1 joule de energia, para que se preserve a rentabilidade almejada. Pode-se, então, calcular $E E$ a partir do valor de $h_{\min }$ (Equação 4) da região considerada e o valor de potência do equipamento empregado, conforme Equação 5.

$$
E E=\frac{h_{\min }}{P}(5)
$$

Segundo a ANEEL (Agência Nacional de Energia Elétrica), a taxa de $\mathrm{kWh}$ mais barata do Brasil é a da concessionária de energia do Rio Grande do Norte (RN), conhecida como COSERN (Companhia energética do Rio grande do Norte), no valor de R\$ 0,37590/ kWh. Considerando-se a cotação do dólar em R\$ 3,93, tem-se, então, o valor de 0,096 USD/kWh. Por fim, pelo Sistema Internacional de Unidades (SI), sabe-se que: 1 watt $=1 \mathrm{~J} / \mathrm{s}$ (Joule/segundo) e, ainda, 1 hora $=3600$ segundos.

Admitindo-se, então, o custo de energia de kWh dado anteriormente e um equipamento que não consuma mais do que 100,0 W e, ainda, substituindo-se os valores do cenário brasileiro na Equação 4, chega-se a um valor de $h_{\min }$ de, aproximadamente, 1863,0 Ghash/s para garantir a lucratividade de 0,10 USD/h. Aplicando-se esse valor de $h_{\min }$ na Equação 5, chega-se, então, ao seguinte resultado: $E E=18,63 \mathrm{Ghash} / \mathrm{J}$.

Considerando-se agora o protocolo Litecoin e um raciocínio análogo ao que acabou de ser descrito para o protocolo Bitcoin, há os seguintes resultados: $h_{\text {min }}=86,37 \mathrm{Mhash} / \mathrm{s}$ e $E E=0,86 \mathrm{Mhash} / \mathrm{J}$, para o país Brasil.

Para analisar o cenário América Latina, escolhem-se os cinco países de maiores PIBs (Produto Interno Bruto) por juntos representarem mais de $80 \%$ do PIB total da América Latina. Os resultados estão nas Figuras 1 e 2. A partir dessas figuras, é possível, imediatamente, perceber que os valores resultantes para o protocolo Bitcoin são bem superiores àqueles obtidos para o protocolo Litecoin. Os valores dos kWh dos países considerados estão na Figura 3. A partir dessa figura, observa-se, imediatamente, que a Argentina possui o menor valor, enquanto a Colômbia o maior. 
Figura 1 - Eficiência energética (mínima) para o protocolo Bitcoin Efficiência energética minima necessária nos 5 maiores PRBs da América Latina para Bitcoin

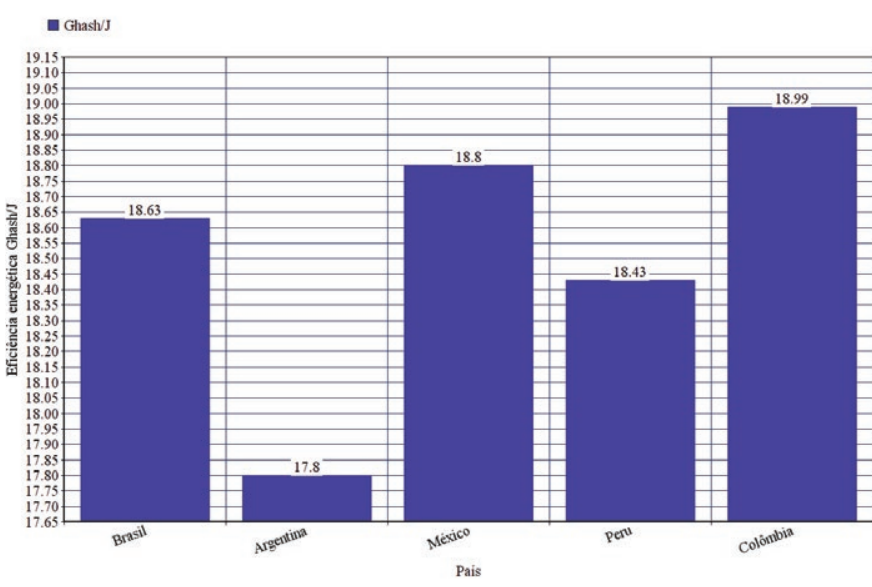

Fonte: Os autores.

Figura 2 - Eficiência energética (mínima) para o protocolo Litecoin. Eficiência energética minima necessária nos 5 maiores PIBs da América Latina para Litecoin

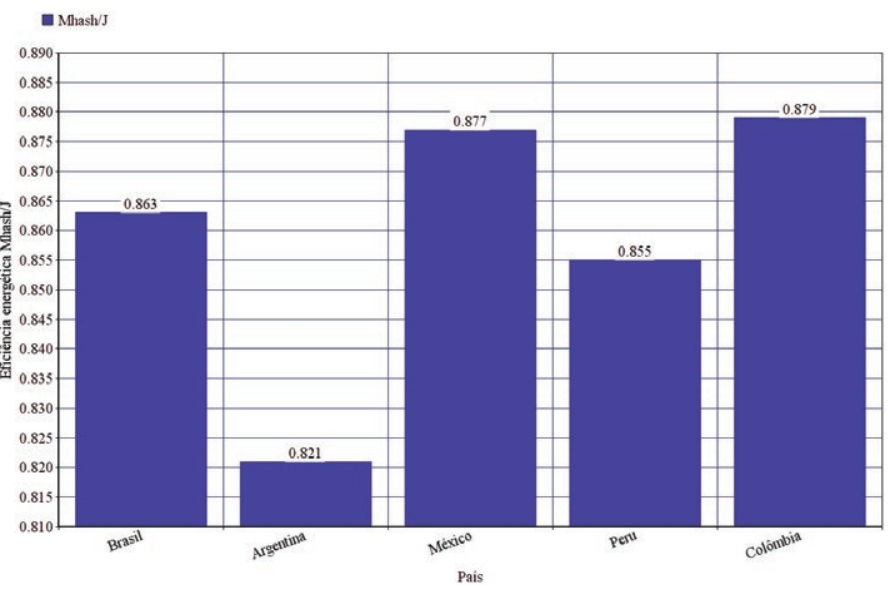

Fonte: Os autores.

Figura 3 - Valores do kWh dos 5 países de maiores PIBs na América Latina Valores em dolares dos $k$ Wh das regốcs conasideradas

$$
\text { Q } \mathrm{kWh}(\mathrm{USD})
$$

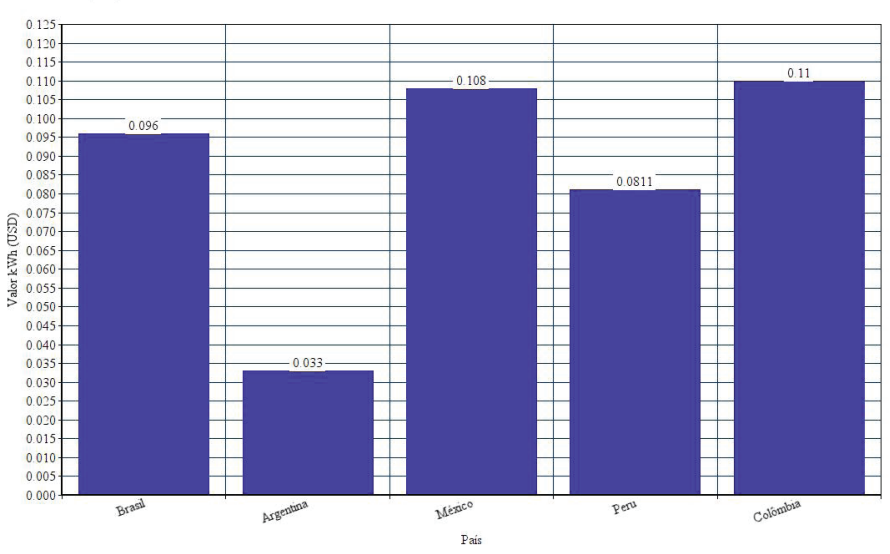

Fonte: Os autores.

Ressalta-se, no entanto, que, para mineração individual, o valor do payout é pago de uma só vez e, apenas, golden nonce é encontrado). Ou seja, apenas ao término da mineração, o minerador recebe seu payout, o qual corresponde, atualmente, a 25 moedas.

Assim, para o estimar o tempo médio em segundos, necessário para encontrar um bloco para ambos protocolos (i.e., Bitcoin e Litecoin), devem, então, ser considerados o valor de $h_{\min }$ e o valor da dificuldade atual $D$, conforme Equação 6.

$$
t=\frac{2^{32} * D}{h_{\min }}(6)
$$

Por meio da Equação 6 e admitindo-se o país Brasil, onde $h_{\min }=1863 \mathrm{Ghash} / \mathrm{s}$ e $D=144116447847$, um bloco do Bitcoin seria, então, resolvido em, aproximadamente, $3,32 \times 10^{8} \mathrm{~s}$. Convertendo-se esse valor em dias, tem-se, aproximadamente, 3.845 dias (ou seja, cerca de 10,53 anos) para se achar um bloco e receber 25 moedas (payout atual). Com a cotação atual, seriam USD 9.588,00 de lucro. Considerando-se agora o mesmo cenário de análise e substituindo-se os valores de $D$ e $h_{\min }$ para o protocolo Litecoin, esse tempo $t$ seria cerca de, apenas, 30 dias, e o valor das 25 moedas seria de USD 77,75.

$\mathrm{O}$ resultado do cálculo anterior alerta para o fato de que, no período de 10 anos, tanto a moeda bitcoin como litecoin teriam passado por dois halvings, o que faria o valor de 25 moedas se tornar um quarto do valor original, ou seja, 6,25 moedas apenas. Nesse sentido, observe que o cálculo de $E E$ proposto não leva em conta os eventos de halvings ao longo do tempo de mineração.

Para efeito de análise com a intenção de evitar-se o evento de halving e, portanto, garantir as 25 moedas de payout, admita o processo de mineração durando no máximo 4 anos (tempo limite para evitar-se o evento de halving), ou seja, um bloco deve ser minerado a cada 1460 dias em média. A partir da manipulação das equações anteriores, é possível mostrar que a $E E$ tem que ser aumentada em, aproximadamente, $263,3 \%$, fazendo a taxa de hash/s do Brasil igual a 4905 Ghash/s (ou o equivalente em $49,05 \mathrm{Ghash} / \mathrm{J}$ de energia).

Observe, ainda, que, ao longo de 10 anos, a dificuldade $D$ e o valor da moeda, relacionadas ao protocolo Bitcoin, podem sofrer variações significativas. Isso faz com que projeções em longo prazo sejam difíceis e geralmente imprecisas. Por outro lado, tendo em vista que, no cenário considerado, o ganho obtido pelo uso do protocolo Litecoin seria alcançado em cerca de apenas 30 dias, as projeções se tornam mais confiáveis e o evento de 
halving não influenciaria o lucro obtido ao final do período estimado para minerar um bloco. Para evidenciar essa imprevisibilidade, a Figura 4 mostra a flutuação da dificuldade $D$ do protocolo Bitcoin no período de março de 2015 até janeiro de 2016.

Figura 4 - Flutuação da dificuldade $D$ do protocolo Bitcoin.

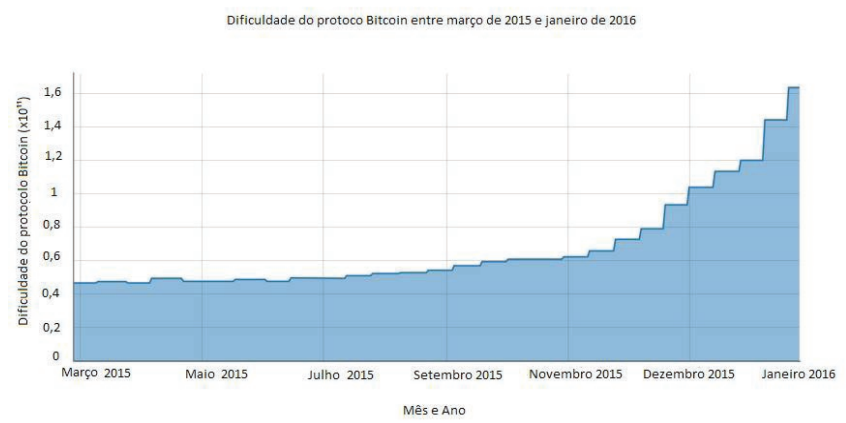

Fonte: Os autores.

Ante as informações e discussões anteriores, os seguintes pontos gerais merecem destaque. Primeiro, o algoritmo de proof-of-work empregado pelo protocolo Litecoin (i.e., algoritmo Scrypt) é mais focado em uso de memória do que em uso de processador. Essa condição permite que equipamentos não necessariamente especializados (i.e., domésticos) possam ser utilizados, com o objetivo de lucro, mais facilmente para a mineração no caso do protocolo Litecoin que no caso do protocolo Bitcoin.

Segundo, o protocolo Litecoin é uma alternativa mais viável para mineração individual, tendo em vista que o tempo para receber a recompensa por bloco minerado (i.e., payout) é bem inferior do que aquele observado para o protocolo Bitcoin. Por isso, o protocolo Litecoin é bem menos susceptível a mudanças, como a variação do preço do kWh e a flutuação da dificuldade de mineração da moeda.

Por fim, a questão central desta pesquisa pode ser satisfatoriamente respondida como segue. É possível afirmar que: mesmo que o valor de cotação atual da moeda litecoin seja inferior àquele da moeda bitcoin, há evidências contundentes que indicam que é mais vantajoso realizar o processo de mineração individual de moedas litecoins que de moedas bitcoins.

\section{Conclusões e trabalhos futuros}

Este artigo teve o objetivo de analisar a rentabilidade econômica da mineração individual de criptomoe- das bitcoins e litecoins. Essa análise foi baseada na avaliação da métrica eficiência energética de cada criptomoeada em cinco países da América Latina, almejando-se uma margem de lucro de 0,10 USD/hora. Três variáveis foram consideradas para o cálculo da métrica: a taxa de processamento de hashes do equipamento, o consumo de energia elétrica do equipamento, e o valor do quilowatt-hora do país da mineração.

Dentre os resultados mais importantes, os seguintes podem ser destacados: (1) para uma mesma rentabilidade econômica do processo de mineração individual, o protocolo Litecoin demanda menor eficiência energética que o protocolo Bitcoin; (2) o processo de mineração do protocolo Bitcoin exige o emprego de hardwares especializados de alto poder de processamento computacional, o que faz com o processo de mineração em pools seja o mais indicado; (3) o processo de mineração do protocolo Litecoin admite o emprego de hardwares domésticos, o que torna bem viável o processo de mineração individual; (4) a mineração individual do protocolo Litecoin tende a ser mais vantajosa que a mineração individual do protocolo Bitcoin.

Por fim, como possível trabalho futuro, sugere-se o estudo de métodos de proof-of-work para o protocolo Bitcoin que não sejam amparados exclusivamente no poder de processamento de hashes dos hardwares utilizados (POON; THADDEUS, 2016). Caso fosse possível reduzir a demanda por poder de processamento computacional, a demanda por energia elétrica, consequentemente, diminuiria, bem como hardwares mais simples poderiam ser utilizados. Isso proporcionaria um maior grau de competitividade ao protocolo Bitcoin frente a protocolos de mesma finalidade.

\section{Referências}

AUMASSON, Jean-Phillipe et al. Cryptanalysis of Dynamic SHA (2). 2009. Disponível em: <https://eprint.iacr. org/2009/184.pdf>. Acesso em: 8 fev. 2016.

BADEV, A.; CHEN, M. Bitcoin: technical background and data analysis. 2014. Disponível em: <http://www.federalreserve.gov/econresdata/feds/2014/files/2014104pap. pdf>. Acesso em: 4 fev. 2016.

BRADBURY, D. Litecoin founder Charles Lee on the origins and potential of the world's second largest cryptocurrency. 2013. Disponível em: <http://www.coindesk.com/ 
litecoin-founder-charles-lee-on-the-origins-and-potential-of-the-worlds-second-largest-cryptocurrency/>. Acesso em: 20 fev. 2016.

DOWD, K.; HUTCHINSON. M. Bitcoin will bite the dust. Cato Journal, v. 35, n. 2. 2015. Disponível em: <http:// object.cato.org/sites/cato.org/files/serials/files/cato-journal/2015/5/cj-v35n2-12.pdf>. Acesso em: 8 fev. 2016.

ESKANDARI, S. et al. A first look at the usability of Bitcoin key management. 2015. Disponível em: <http://www.internetsociety.org/sites/default/files/05_3_3.pdf>. Acesso em: 9 fev. 2016.

WHITE, L. H. The market for cryptocurrencies. Cato Journal, v. 35, n. 2, 2015. Disponível em: <http://object.cato.org/sites/cato.org/files/serials/files/cato-journal/2015/5/cj-v35n2-13.pdf>. Acesso em: 2 fev. 2016.

LUTHER, W. J. Bitcoin and the future of digital payments. 2015. Disponível em: <http://ssrn.com/abstract $=2631314>$. Acesso em: 10 fev. 2016.

MURPHY, R. P. The economics of Bitcoin. Library and Liberty. 2013. Disponível em: <www.econlib.org/library/ Columns/y2013/Murphybitcoin.html>. Acesso em: 10 fev. 2016.

NAKAMOTO, Satoshi. Bitcoin: a peer-to-peer electronic cash system. 2008. Disponível em: <https://bitcoin.org/ bitcoin.pdf $>$. Acesso em: 30 dez. 2015.
NIELSEN, M. How the Bitcoin protocol actually works. 2013. Disponível em: <http://www.michaelnielsen.org/ ddi/how-the-bitcoin-protocol-actually-works/>. Acesso em: 9 fev. 2016.

PERCIVAL, C. Stronger key derivation via sequential memory-hard functions. 2009. Disponível em: <http:// www.tarsnap.com/scrypt/scrypt.pdf>. Acesso em: 30 jan. 2016.

POON, J.; THADDEUS, D. The Bitcoin lightning network: acalable off-chain instant payments. 2016. Disponível em: <http://lightning.network/lightning-network-paper. pdf $>$. Acesso em: 25 jan. 2016.

ROSENFIELD, Meni. Analysis of Bitcoin pooled mining reward systems. 2011. Disponível em: <https://bitcoil. co.il/pool_analysis.pdf>. Acesso em: 30 jan. 2016.

CHÁVEZ, J. J. G.; RODRIGUES, C. K. S. Hopping among pools in the Bitcoin mining network. The SIJ Transactions on Computer Networks \& Communication Engineering (CNCE), v. 3, n. 2, 2015.

PAZMIÑO, J. E.; RODRIGUES, C. K. S. Simply dividing a Bitcoin network node may reduce transaction verification time. The SIJ Transactions on Computer Networks \& Communication Engineering (CNCE), v. 3, n. 2, 2015. 